\title{
Boundary Galerkin Method of a Skew-Derivative Problem in the Exterior of an Open Arc Based on Chebyshev Polynomials
}

\author{
Wei Sun ${ }^{1,2}$ and Fuming $\mathrm{Ma}^{1}$ \\ ${ }^{1}$ School of Mathematics, Jilin University, Changchun 130012, China \\ ${ }^{2}$ Harbin University of Science and Technology, Harbin 150080, China \\ Correspondence should be addressed to Wei Sun; mastersunwei@gmail.com
}

Received 14 December 2012; Accepted 23 January 2013

Academic Editor: Oleg V. Gendelman

Copyright (C) 2013 W. Sun and F. Ma. This is an open access article distributed under the Creative Commons Attribution License, which permits unrestricted use, distribution, and reproduction in any medium, provided the original work is properly cited.

\begin{abstract}
A problem modeling Hall effect in a semiconductor film from an electrode of arbitrary shape is considered, which is a skewderivative problem. Boundary Galerkin method for solving the problem in Sobolev spaces is developed firstly. The solution is represented in the form of the combined angular potential and single-layer potential. The final integral equations do not contain hypersingular integrals. Uniqueness and existence of the solution to the equations are proved. The weakly singular and Cauchy singular integral arising in these equations can be computed directly by truncated series of Chebyshev polynomials with their weighting function without approximation. The numerical simulation showing the high accuracy of the scheme is presented.
\end{abstract}

\section{Introduction}

Hall effect is the production of a voltage difference across a semiconductor, transverse to an electric current in the conductor and a magnetic field perpendicular to the current. It is widely used in industrial automation, detection, and information processing, which is the basic method for the study of semiconductor material properties. In this paper, we concern an electric current in a semiconductor coming from an electrode, which can be modeled by an open arc. A magnetic is orthogonal to the film. The directions of electric current and electric intensity are not the same because of Hall effect. From a mathematical standpoint, the problem is a skew-derivative problem in the exterior of an open arc in a plane.

The boundary integral has been a means of solving problems in the exterior of an open arc. The difficulty of the problem is that the solution is continuous at the tips, but its gradient may have weak singularity. Numerical methods of problems are considered in Hölder space in a series of papers [1-3]. The analysis in [4] is performed within a Sobolev space setting and a weak solution concept, but no numerical method is presented. These authors construct the solution, via a combination of a single- and a double-layer potential, which can not describe the singularity at the tips directly. Thus, another more difficult problem is introduced, that is, hypersingularity.

In [5], the author constructs the solution by singlelayer potential and nonclassical angular potential [6-10] and proves the existence and uniqueness of the solution. An angular potential can be seen as a double layer potential under certain conditions. It has the same order of singularity as a single-layer potential, which is different from a double-layer potential. This makes the final integral system much simpler. The solution which is a combination of a single-layer and angular potential may have weak singularity at the tips of arc if the density function has the form $\widehat{\mu} / \sqrt{1-\sigma^{2}}$.

In [11], a numerical method for solving this problem is first considered. It numerically solved a Fredholm integral equation of the second kind in Hölder space. The method can avoid the hypersingularity caused from the doublelayer potential. But due to Cauchy singular integral of the system and the special form of density function, approximate calculation of singular integral can not be avoided. In recent years, the inverse problems for arc have been considered by 
some researchers. In [12-14], the authors obtain asymptotic analysis in terms of the size of the solutions to scattering problems by cracks. Such asymptotics are useful for solving inverse and imaging problems.

In the present paper, we consider the problem in Sobolev spaces firstly. The equations which consist of a Cauchy integral equation of the first kind with additional condition are derived from summing up a single-layer potential and an angular potential. We discuss the uniqueness and existence of the solution to boundary Galerkin method. The purpose of this paper is to develop an efficient solution method. Due to the properties of Chebyshev polynomials, several researchers $[1,3]$ use them for solving integral equations. In this paper, we approximate the unknown function by means of truncated series of Chebyshev polynomials with their weighting function. Thus, the numerical solution may have weak singularity at the tips of the arc and the singular integral can be computed directly. The convergence is proved. Some numerical examples are presented in order to test the method by comparison with explicit solutions and the numerical solutions of [11].

The plan of the paper is as follows: in Section 2, model problem is listed. We introduce our formulation of skewderivative problem and proceed by establishing the existence and uniqueness of a solution to integral equations. Then in Section 3, we develop a spectral boundary element method for solving the integral equations numerically and discuss some numerical techniques. Section 4 presents several numerical experiments to illustrate the accuracy.

\section{Uniqueness and Existence}

In this section, we introduce the model problem firstly. Thus the problem is reduced to an integral equation with some additional conditions. Then we establish the uniqueness and existence of the solution to the integral equations in Sobolev spaces, by inversion of the integral operator.

Consider a constant magnetic field which is perpendicular to a plane semiconductor film $x=\left(x_{1}, x_{2}\right) \in \mathbb{R}^{2}$. An electrode is placed in the unbounded film. The electrode is modeled by an open curve $\Gamma \in C^{2, \lambda}, \lambda \in(0,1]$. Set

$$
\Gamma:=\left\{y=y(s)=\left(y_{1}(s), y_{2}(s)\right): s \in[-1,1]\right\} .
$$

By $z_{-1}, z_{1}$ we denote the two end points $z_{1}:=y(1), z_{-1}:=$ $y(-1)$ of $\Gamma$. Denote the left-hand of $\Gamma$ by $\Gamma^{+}$when $s$ increases, while the opposite side is called $\Gamma^{-}$. The outward normal of $\Gamma^{+}$is denoted by $n$. The tangent direction $\tau$ of $\Gamma$ is directed into the direction of $s$ increasing. Obviously, $n(x(s))=\left(\dot{x}_{2}(s),-\dot{x}_{1}(s)\right) /|\dot{x}(s)|=\dot{x}(s)^{\perp} /|\dot{x}(s)|$, where $|\dot{x}(s)|:=\sqrt{\dot{x}_{1}(s)^{2}+\dot{x}_{2}(s)^{2}}$.

The electric-field potential $u$ is the solution of the skewderivative problem as follows:

$$
\begin{gathered}
\Delta u=0, \quad \mathbb{R}^{2} \backslash \Gamma, \\
\frac{\partial}{\partial n_{x}} u(x(s))+\beta \frac{\partial}{\partial \tau_{x}} u(x(s))=f(s), \quad \Gamma,
\end{gathered}
$$

$$
\begin{gathered}
|u|=O(1), \quad|x| \longrightarrow \infty, \\
|\nabla(u)|=o\left(|x|^{-1}\right), \quad|x| \longrightarrow \infty,
\end{gathered}
$$

where $\beta=\alpha M, \alpha$ is the mobility of the carriers, and $M$ is the projection of the magnetic induction onto $x_{3}$ axis. Suppose $\beta$ is a real constant. The electric current satisfies the equations as follows:

$$
\operatorname{div} \mathbf{J}=0, \quad \mathbf{J}=\Lambda \mathbf{E}, \quad \mathbf{E}=-\nabla u,
$$

where

$$
\Lambda=\frac{\eta}{1+\beta^{2}}\left(\begin{array}{cc}
-1 & \beta \\
-\beta & 1
\end{array}\right)
$$

and $\eta$ is a constant. It is easy to see that the direction of current density $\mathbf{J}$ and the intensity of the electric field $\mathbf{E}$ do not coincide if $\beta \neq 0$. These equations lead to the first formula of (2). The boundary condition on $\Gamma$ in (2) is deduced by

$$
\left(\mathbf{J}, n_{x}\right)_{x \in \Gamma}=\frac{\eta}{1+\beta^{2}} f(s) .
$$

Problem (2) is considered by [5]. The author establishes the existence of a solution by a combined single-layer potential and angular potential approach in Hölder space and proves uniqueness up to arbitrary constant.

Consider the angular potential introduced (see [6]):

$$
v[\mu](x)=-\frac{1}{2 \pi} \int_{\Gamma} \mu(\sigma) V(x, \sigma) d s_{\sigma},
$$

where $V(x, \sigma)$ is defined by

$$
\cos V(x, \sigma)=\frac{x_{1}-y_{1}(\sigma)}{|x-y(\sigma)|}, \quad \sin V(x, \sigma)=\frac{x_{2}-y_{2}(\sigma)}{|x-y(\sigma)|}
$$

From the definition, it is easy to see that an angular potential is a multivalued function. In order to make it single-valued, it is necessary to require the following additional condition (see [6]):

$$
\int_{\Gamma} \mu d s=0
$$

Integrating it by parts, $v[\mu](x)$ becomes a double-layer potential as follows:

$$
v[\mu](x)=\frac{1}{2 \pi} \int_{\Gamma} \rho[\mu] \frac{\partial}{\partial n_{y}} \ln |x-y| d s_{y},
$$

where

$$
\rho[\mu]=\int_{\Gamma_{\sigma}} \mu d s
$$

and $\Gamma_{\sigma}:=\left\{y(s)=\left(y_{1}(s), y_{2}(s)\right), s \in[-1, \sigma]\right\}$. 

form:

In [5], the solution of (2) is constructed in the following

$$
u[\mu](x)=v[\mu](x)-\beta w[\mu](x)+C,
$$

where $C$ is an arbitrary constant. $w$ is the single-layer potential

$$
w[\mu](x)=\frac{1}{2 \pi} \int_{\Gamma} \mu(\sigma) \ln |x-y| d s_{y} .
$$

If $\mu(\sigma)$ in (11) behaves as $\widehat{\mu} / \sqrt{1-\sigma^{2}}$, where $\widehat{\mu}$ is a continuous function, the solution $u[\mu](x)$ has singularity at the endpoints of $\Gamma$, that is,

$$
|\nabla u(x)| \leq C|x-x(d)|^{\epsilon},
$$

with $d= \pm 1$ and $\epsilon \in(-1,0]$.

We put (11) in the boundary condition of (2) and get the following integral equation:

$$
-\frac{1+\beta^{2}}{2 \pi} \int_{\Gamma} \mu(\sigma) \frac{\sin \varphi_{0}(y(s), y(\sigma))}{|y(s)-y(\sigma)|} d s_{\sigma}=f(s),
$$

where $\varphi_{0}(x, y)$ is the angle between the vector $\overrightarrow{x y}$ and the direction of the normal $n_{y}$. If $\mu$ in (11) satisfies (14), it is the solution of problem (2).

First, we extend the arc $\Gamma$ to a piecewise smooth, simple connected, closed curve $\partial D$ and define the following spaces (see $[15])$ :

$$
\begin{gathered}
L^{2}(\Gamma):=\left\{\left.u\right|_{\Gamma}: u \in L^{2}(\partial D)\right\}, \\
H^{1 / 2}(\Gamma):=\left\{\left.u\right|_{\Gamma}: u \in H^{1 / 2}(\partial D)\right\}, \\
\widetilde{H}^{1 / 2}(\Gamma):=\left\{\left.u\right|_{\Gamma}: u \in H^{1 / 2}(\partial D): \operatorname{supp} u \subseteq \bar{\Gamma}\right\},
\end{gathered}
$$

where $L^{2}(\partial D), H^{1 / 2}(\partial D)$ denote the usual Sobolev spaces. That is to say, $\widetilde{H}^{1 / 2}(\Gamma)$ contains functions $u \in H^{1 / 2}(\Gamma)$ such that their extension by zero to $\partial D$ is in $H^{1 / 2}(\partial D)$. In [16], the author characterizes $\widetilde{H}^{1 / 2}(\Gamma)$ by using the norm

$$
\|u\|_{H_{00}^{1 / 2}}=\left\{\|u\|_{H^{1 / 2}(\Gamma)}^{2}+\left\|\rho^{-1 / 2} u\right\|_{L^{2}(\Gamma)}^{2}\right\}^{1 / 2},
$$

where $\rho=\operatorname{dist}\left(x, z_{ \pm 1}\right)$. It is shown that the norms

$$
\|u\|_{H_{00}^{1 / 2}(\Gamma)},\|\widetilde{u}\|_{H^{1 / 2}(\partial D)} \quad \text { with } \widetilde{u}= \begin{cases}u & \text { for } x \in \Gamma, \\ 0 & \text { otherwise. }\end{cases}
$$

are equivalent.

Now define the dual space of $\widetilde{H}^{1 / 2}(\Gamma)$ by $H^{-1 / 2}(\Gamma)$ and the dual space of $H^{1 / 2}(\Gamma)$ by $\widetilde{H}^{-1 / 2}(\Gamma)$. Hence we have the chain

$$
\widetilde{H}^{1 / 2}(\Gamma) \subset H^{1 / 2}(\Gamma) \subset L^{2}(\Gamma) \subset \widetilde{H}^{-1 / 2}(\Gamma) \subset H^{-1 / 2}(\Gamma) .
$$

Note that $\widetilde{H}^{-1 / 2}(\Gamma)$ also coincides with $H_{\bar{\Gamma}}^{-1 / 2}(\partial D):=\{u \in$ $H^{-1 / 2}(\partial D)$ : supp $\left.u \subset \bar{\Gamma}\right\}$.
In order to prove the existence and uniqueness of solutions for (8) and (14), we introduce two boundary integral operators

$$
T: \widetilde{H}^{1 / 2}(\Gamma) \longrightarrow H^{-1 / 2}(\Gamma), \quad I: V \longrightarrow \widetilde{H}^{1 / 2}(\Gamma),
$$

which are defined by

$$
\begin{gathered}
T \psi=\frac{\partial}{\partial n_{x}} \int_{\Gamma} \psi(y) \frac{\partial}{\partial n_{y}} \ln |x-y| d s_{y}, \\
I \mu=\int_{\Gamma_{\sigma}} \mu d s,
\end{gathered}
$$

with $V=\left\{\mu \in \widetilde{H}^{-1 / 2}(\Gamma): \int_{\Gamma} \mu d s=0\right\}$. It is easy to see $T \circ I$ is the limit of normal derivative of angular potential on $\Gamma$.

Lemma 1. Under the condition of (8)

$$
\begin{aligned}
& \lim _{x \rightarrow x(s) \in \Gamma} \frac{\partial v[\mu](x)}{\partial n_{x}} \\
& \quad=-\frac{1}{2 \pi} \int_{-1}^{1} \mu(\sigma) \frac{\sin \varphi_{0}(x(s), y(\sigma))}{|x(s)-y(\sigma)|}|\dot{y}(\sigma)| d \sigma .
\end{aligned}
$$

One rewrites (14) with (8) in operator form

$$
\left(1+\beta^{2}\right) T I \mu=f .
$$

Lemma 2. I has a bounded inverse operator.

Proof. Let $\mu d s=f d t$. It is easy to see $f \in \widetilde{H}^{-1 / 2}[-1,1]$ and $\int_{-1}^{1} f d t=0$. Let $F=I f$. Obviously, $F \in \widetilde{H}^{1 / 2}(\Gamma)$ and $F\left(z_{ \pm 1}\right)=0$. There is a homeomorphism from $\widetilde{H}^{1 / 2}(\Gamma)$ to $\widetilde{H}^{1 / 2}[0,2 \pi]$. Define an operator $D: D\left(e^{\mathrm{imt}}\right)=\mathrm{ime}^{\mathrm{imt}}$. Thus $D$ is the bounded inverse operator of $I$.

It is known that $-T$ is positive and bounded below up to a compact perturbation, that is, there exists a compact operator

$$
L: \widetilde{H}^{1 / 2}(\Gamma) \longrightarrow H^{-1 / 2}(\Gamma)
$$

such that

$$
\operatorname{Re}\langle-(T+L) \psi, \bar{\psi}\rangle \geq C\|\psi\|^{2}, \quad \text { for } \psi \in \widetilde{H}^{1 / 2}(\Gamma),
$$

where $\langle\cdot, \cdot\rangle$ denotes the duality pairing between $H^{-1 / 2}(\Gamma)$ and $\widetilde{H}^{1 / 2}(\Gamma)$. In other words, $T$ is a Fredholm operator with zero index. It can be easily proved that the homogeneous equation (14) has only a trivial solution. As noted above, we arrive at the following assertion.

Theorem 3. Equation (22) has a unique solution $\mu \in \widetilde{H}^{-1 / 2}(\Gamma)$ for any $f \in H^{-1 / 2}(\Gamma)$.

\section{Spectral Boundary Galerkin Method}

We approximate the unknown function $\mu(\sigma)$ in (22) by truncated series of Chebyshev polynomials with weighting 
function $1 / \sqrt{1-\sigma^{2}}$. As mentioned above, the solution may have weak singularity. Let $T_{n}(\sigma)$ and $U_{n}(\sigma)$ denote the Chebyshev polynomials of first and second kinds

$$
\begin{gathered}
T_{n}(\sigma)=\cos (n \arccos \sigma), \\
U_{n}(\sigma)=\frac{\sin ((n+1) \arccos \sigma)}{\sin (\arccos \sigma)}
\end{gathered}
$$

with real values over $[-1,1]$. Then Chebyshev polynomials are orthogonal with respect to the weighting functions

$$
\begin{aligned}
& \int_{-1}^{1} T_{n}(\sigma) T_{m}(\sigma) \frac{1}{\sqrt{1-\sigma^{2}}} d \sigma= \begin{cases}0 & n \neq m, \\
\frac{\pi}{2} & n=m=0, \\
\pi & n=m \neq 0,\end{cases} \\
& \int_{-1}^{1} U_{n}(\sigma) U_{m}(\sigma) \sqrt{1-\sigma^{2}} d \sigma= \begin{cases}0 & n \neq m, \\
\frac{\pi}{2} & n=m=0 .\end{cases}
\end{aligned}
$$

Next we construct the approximation spaces:

$$
\begin{aligned}
& \mathbb{Q}_{N}=\operatorname{span}\left\{\frac{1}{\sqrt{1-\sigma^{2}}} T_{n}(\sigma)\right\}_{n=1}^{N}, \\
& \mathbb{P}_{N}=\operatorname{span}\left\{\sqrt{1-\sigma^{2}} U_{n}(\sigma)\right\}_{n=0}^{N} .
\end{aligned}
$$

Let us show approximation property over the associated Sobolev space.

Lemma 4. (1) The space $\mathbb{P}_{N}$ is a closed subspace of $\widetilde{H}^{1 / 2}[-1,1]$.

(2) $\mathbb{P}_{\infty}=\lim _{N \rightarrow \infty} \mathbb{P}_{N}$ is dense in $\widetilde{H}^{1 / 2}[-1,1]$.

Proof. Obviously, $\sqrt{1-\sigma^{2}} U_{n}(\sigma)$ belongs to $\widetilde{H}^{1 / 2}(\Gamma)$. For $f \in$ $\widetilde{H}^{1 / 2}[-1,1], \exists \psi \in C_{0}^{\infty}(-1,1)$, s.t.

$$
\|f-\psi\| \leq \varepsilon
$$

According to the Weierstrass approximation theorem, there is an integer $M>0$ such that

$$
\max _{x \in[-1,1]}\left|\psi-p_{M}\right|<\varepsilon
$$

where $p_{M}$ is the Bernstein polynomial of order $M$. On the basis of the definition of $p_{M}$ and $\psi(-1)=\psi(1)=0$, we have

$$
p_{M}(-1)=p_{M}(1)=0 \text {. }
$$

Take the transform

$$
p_{M}=\sqrt{1-x^{2}} p^{\prime}
$$

Obviously, $p^{\prime} \in C[-1,1]$. So there is a polynomial $P$ such that

$$
\max _{x \in[-1,1]}\left|p^{\prime}-P\right|<\varepsilon
$$

Thus

$$
\begin{aligned}
\left\|f-\sqrt{1-x^{2}} P\right\| \leq & \|f-\psi\|+\left\|\psi-\sqrt{1-x^{2}} P\right\| \\
\leq & \|f-\psi\| \\
& \quad+\max \left\{\left|\psi-p_{M}\right|+\sqrt{1-x^{2}}\left|p^{\prime}-P\right|\right\} \\
\leq & 3 \varepsilon .
\end{aligned}
$$

The proof is complete.

Obviously, the next Lemma follows.

Lemma 5. (1) The space $\mathbb{Q}_{N}$ is a closed subspace of $\widetilde{H}^{-1 / 2}[-1,1]$.

(2) $\mathbb{Q}_{\infty}=\lim _{N \rightarrow \infty} \mathbb{Q}_{N}$ is dense in $\widetilde{H}^{-1 / 2}[-1,1]$.

The idea of constructing the finite element space is to describe $\mu$ through the truncated expansion:

$$
\varphi_{N}(\sigma)=\sum_{n=1}^{N} c_{n} \frac{1}{\sqrt{1-\sigma^{2}}} T_{n}(\sigma)
$$

The spectral Galerkin variational formulation of problem (22) is to find $\varphi_{N} \in \mathbb{Q}_{N}$, and $\int_{-1}^{1} \varphi_{N}(\sigma)|\dot{y}(\sigma)| d \sigma=0$,

$$
\left(1+\beta^{2}\right)\left\langle T I \varphi_{N}, \psi\right\rangle=\langle f, \psi\rangle, \quad \forall \psi \in \mathbb{P}_{N-1}
$$

Thanking [17], the following estimation holds:

$$
\left\|\mu-\varphi_{N}\right\|_{\widetilde{H}^{-1 / 2}} \leq C \inf _{\psi_{N} \in \mathbb{Q}_{N}}\left\|\mu-\psi_{N}\right\|_{\widetilde{H}^{-1 / 2}} .
$$

By using of the relation (22) and (14) and the finite dimensional subspace introduced above, we can rewrite the spectral Galerkin method as to find $c_{n}, n=1, \ldots, N$, such that

$$
\begin{gathered}
-\frac{\left(1+\beta^{2}\right)}{2 \pi} \sum_{n=1}^{N} c_{n} \int_{-1}^{1} \sqrt{1-s^{2}} U_{m}(s)|\dot{y}(s)| \\
\times\left\{\int_{-1}^{1} \frac{T_{n}(\sigma)}{\sqrt{1-\sigma^{2}}} \frac{\sin \varphi_{0}(y(s), y(\sigma))}{|y(s)-y(\sigma)|}|\dot{y}(\sigma)| d \sigma\right\} d s \\
=\int_{-1}^{1} \sqrt{1-s^{2}} U_{m}(s) f(s)|\dot{y}(s)| d s, \\
m=0,1 \ldots, M,
\end{gathered}
$$

$$
\sum_{n=1}^{N} c_{n} \int_{-1}^{1} \frac{T_{n}(\sigma)}{\sqrt{1-\sigma^{2}}}|\dot{y}(\sigma)| d \sigma=0
$$

It can be shown that

$$
\frac{\sin \varphi_{0}(y(s), y(\sigma))}{|y(s)-y(\sigma)|}-\frac{1}{(\sigma-s)|\dot{y}(\sigma)|} \in C([-1,1] \times[-1,1]) .
$$


Rewrite (37) in the form

$$
\begin{aligned}
& \sum_{n=1}^{N} c_{n} \int_{-1}^{1} \sqrt{1-s^{2}} U_{m}(s)|\dot{y}(s)| \\
& \quad \times\left(\int_{-1}^{1} \frac{T_{n}(\sigma)}{\sqrt{1-\sigma^{2}}(\sigma-s)} d \sigma\right) d s \\
& +\sum_{n=1}^{N} c_{n} \int_{-1}^{1} \sqrt{1-s^{2}} U_{m}(s)|\dot{y}(s)| \\
& \quad \times\left\{\int_{-1}^{1}\left(\frac{\sin \varphi_{0}(y(s), y(\sigma))}{|y(s)-y(\sigma)|}-\frac{1}{(\sigma-s)|\dot{y}(\sigma)|}\right)\right. \\
& \left.\quad \times \frac{T_{n}(\sigma)}{\sqrt{1-\sigma^{2}}}|\dot{y}(\sigma)| d \sigma\right\} \\
& \quad=\frac{2 \pi}{1+\beta^{2}} \int_{-1}^{1} \sqrt{1-s^{2}} U_{m}(s) f(s)|\dot{y}(s)| d s .
\end{aligned}
$$

It is convenient to evaluated integrals in (40) by using the Gauss-Chebyshev integration rule of the first kind

$$
\int_{-1}^{1} \frac{1}{\sqrt{1-\sigma^{2}}} f(\sigma) d \sigma \approx \frac{\pi}{J} \sum_{j=1}^{J} f\left(\alpha_{j}\right)
$$

with $\alpha_{j}=\cos \{(2 j-1) \pi / 2 J\}, j=1, \ldots, J$, and the second kind

$$
\int_{-1}^{1} \sqrt{1-\sigma^{2}} f(\sigma) \approx \frac{\pi}{J+1} \sum_{j=1}^{J} f\left(\beta_{j}\right) \sin ^{2} \frac{j \pi}{J+1}
$$

where $\beta_{j}=\cos (j \pi /(J+1)), j=1, \ldots, J$.

For the first integral in (40), it is Cauchy intergal and the following formula is useful:

$$
\int_{-1}^{1} \frac{T_{n}(\sigma)}{(\sigma-s) \sqrt{1-\sigma^{2}}} d \sigma=\pi U_{n-1}(s), \quad n \geq 1 .
$$

If $|\dot{y}(s)| \neq$ const, $M=N-2$, the integral can be evaluated by (42):

$$
\begin{gathered}
\int_{-1}^{1} \sqrt{1-s^{2}} U_{m}(s)|\dot{y}(s)|\left(\int_{-1}^{1} \frac{T_{n}(\sigma)}{\sqrt{1-\sigma^{2}}(\sigma-s)} d \sigma\right) d s \\
\approx \frac{\pi^{2}}{J+1} \sum_{j=1}^{J} U_{m}\left(\beta_{j}\right) U_{n-1}\left(\beta_{j}\right)\left|\dot{y}\left(\beta_{j}\right)\right| \sin ^{2} \frac{j \pi}{J+1} .
\end{gathered}
$$

In addition, there is an important condition (8), which can also be computed by (41):

$$
\frac{\pi}{J} \sum_{n=1}^{N} c_{n} \sum_{j=1}^{J} T_{n}\left(\alpha_{j}\right)\left|\dot{y}\left(\alpha_{j}\right)\right|=0
$$

If $|\dot{y}(s)|=C, M=N-1$, (8) can be satisfied naturally according to the definition of $\mathbb{Q}_{N}$. We have

$$
\begin{aligned}
& \int_{-1}^{1} \sqrt{1-s^{2}} U_{m}(s)|\dot{y}(s)|\left(\int_{-1}^{1} \frac{T_{n}(\sigma)}{\sqrt{1-\sigma^{2}}(\sigma-s)} d \sigma\right) d s \\
& \quad= \begin{cases}0, & n \neq m+1, \ldots \\
\frac{\pi^{2}}{2} C, & n=m+1, \ldots\end{cases}
\end{aligned}
$$

which is obtained by directly using of the orthogonal relation of Chebyshev polynomials $U_{m}$.

Assume that the stiffness matrix constructed by the first term in (40) is denoted by $I$. In the second case, $I$ is a diagonal matrix

$$
I_{N \times N}=\left(\begin{array}{cccc}
\frac{\pi^{2}}{2} C & & & \\
& \frac{\pi^{2}}{2} C & & \\
& & \ddots & \\
& & & \frac{\pi^{2}}{2} C
\end{array}\right) .
$$

Consider the computation of the second term of (40). The contribution for stiffness matrix from the second term in (40) is denoted by $A=\left\{a_{i j}\right\}_{N \times N}$. According to (41) and (42), the entries of matrix $A$

$$
\begin{aligned}
a_{m+1, n} \approx & \frac{\pi^{2}}{J(J+1)} \sum_{i=1}^{J} \sum_{j=1}^{J} U_{m}\left(\beta_{i}\right) T_{n}\left(\alpha_{j}\right)\left|\dot{y}\left(\alpha_{j}\right)\right| \\
& \times\left[\frac{\sin \varphi_{0}\left(y\left(\beta_{i}\right), y\left(\alpha_{j}\right)\right)}{\left|y\left(\beta_{i}\right)-y\left(\alpha_{j}\right)\right|}-\frac{1}{\left(\alpha_{j}-\beta_{i}\right)\left|\dot{y}\left(\alpha_{j}\right)\right|}\right] \\
& \times\left|\dot{y}\left(\beta_{i}\right)\right| \sin ^{2} \frac{i \pi}{J+1} .
\end{aligned}
$$

Therefore, the stiffness matrix for (40) is $I+A$. The third term can also be evaluated by (42):

$$
\begin{aligned}
\int_{-1}^{1} & \sqrt{1-\sigma^{2}} U_{m}(s) f(s)|\dot{y}(s)| d s \\
& \approx \frac{\pi}{J+1} \sum_{j=1}^{J} f\left(\beta_{j}\right) U_{m}\left(\beta_{j}\right) \sin ^{2} \frac{j \pi}{J+1}\left|\dot{y}\left(\beta_{j}\right)\right| .
\end{aligned}
$$

Finally, we get a linear algebraic system of equations with respect to $C=\left\{c_{1}, c_{2}, \ldots, c_{N}\right\}$.

Next, we recover single-layer potential $w[\mu]$ and angular potential $v[\mu](x)$ by $(41)$ :

$$
w[\mu](x) \approx \frac{1}{2 J} \sum_{j=1}^{J} \sqrt{1-\alpha_{j}^{2}} \mu\left(\alpha_{j}\right) \ln \left|x-y\left(\alpha_{j}\right)\right|\left|\dot{y}\left(\alpha_{j}\right)\right|,
$$

$$
v[\mu](x) \approx \frac{1}{2 J} \sum_{j=1}^{J} \sqrt{1-\alpha_{j}^{2}} \mu\left(\alpha_{j}\right) V\left(x, \alpha_{j}\right)\left|\dot{y}\left(\alpha_{j}\right)\right| .
$$


TABLE 1: $L^{2}$-errors for a straight line case with $\beta \equiv 1, f \equiv 1(W, V$ with $M=160, h=0.0125$ and $w, v$ with $J=160, N=10)$.

\begin{tabular}{lcccc}
\hline$d$ & $\|w-\widetilde{w}\|_{L^{2}}$ & $\|W-\widetilde{w}\|_{L^{2}}$ & $\|v-\widetilde{v}\|_{L^{2}}$ & $\|V-\widetilde{v}\|_{L^{2}}$ \\
\hline $10^{-1}$ & $3.2292 \times 10^{-16}$ & $1.4964 \times 10^{-4}$ & $3.5929 \times 10^{-16}$ & $6.1281 \times 10^{-4}$ \\
$10^{-2}$ & $2.5694 \times 10^{-5}$ & $1.7546 \times 10^{-4}$ & $2.6125 \times 10^{-5}$ & $1.3991 \times 10^{-3}$ \\
$10^{-3}$ & $1.1000 \times 10^{-3}$ & $1.7902 \times 10^{-4}$ & $9.7890 \times 10^{-4}$ & $3.2491 \times 10^{-3}$ \\
\hline
\end{tabular}

TABLE 2: $L^{2}$-errors for an arc on the unite circle case with $\beta \equiv 1$, $f \equiv 1$.

\begin{tabular}{lccc}
\hline$J$ & $N=3$ & $N=5$ & $N=10$ \\
\hline 20 & $6.66271 \times 10^{-6}$ & $6.61987 \times 10^{-8}$ & $5.20990 \times 10^{-12}$ \\
40 & $6.48476 \times 10^{-6}$ & $6.41134 \times 10^{-8}$ & $5.40632 \times 10^{-12}$ \\
80 & $6.43437 \times 10^{-6}$ & $6.32523 \times 10^{-8}$ & $5.33493 \times 10^{-12}$ \\
\hline
\end{tabular}

TABLE 3: An arc on the unit circle of $[11]: \beta \equiv 1, f \equiv 1$.

\begin{tabular}{lccc}
\hline & $M=20$ & $M=40$ & $M=80$ \\
\hline Errors & $1.0027 \times 10^{-3}$ & $3.6568 \times 10^{-4}$ & $1.31818 \times 10^{-4}$ \\
\hline
\end{tabular}

TABLE 4: $L^{2}$-errors for an arc on the unite circle case with $\beta \equiv 1$, $f \equiv \cos (x)+\sin (x)$.

\begin{tabular}{lccc}
\hline$J$ & $N=3$ & $N=5$ & $N=10$ \\
\hline 20 & $2.0634 \times 10^{-2}$ & $2.6524 \times 10^{-4}$ & $2.54695 \times 10^{-10}$ \\
40 & $2.01549 \times 10^{-2}$ & $2.56218 \times 10^{-4}$ & $2.63766 \times 10^{-10}$ \\
80 & $1.9999 \times 10^{-2}$ & $2.53127 \times 10^{-4}$ & $2.61626 \times 10^{-10}$ \\
\hline
\end{tabular}

TABLE 5: An arc on the unit circle of [11]: $\beta \equiv 1, f \equiv \cos (x)+\sin (x)$.

\begin{tabular}{cccc}
\hline & $M=20$ & $M=40$ & $M=80$ \\
\hline Errors & $2.4832 \times 10^{-3}$ & $8.7919 \times 10^{-4}$ & $3.1122 \times 10^{-4}$ \\
\hline
\end{tabular}

It is easy to see that we can get approximate electric-field potential $u$ by

$$
u(x)=v[\mu](x)-\beta w[\mu](x)+C .
$$

Although (6) can also be expressed by a double-layer potential, such an expression has more strong singularity near $\Gamma$ compared with (6). So (51) is deduced by (6).

\section{Numerical Examples}

In Section 3 we develop a method for finding the numerical solution of the integral equation (22). More precisely, a spectral Galerkin method is used. We reduce it to a linear algebraic system. In this section, we describe the results of the numerical tests mentioned above. We examine the feasibility of the numerical method for solving (22) and compare the numerical solution with these solutions of [11] for certain function $f$ on the right-hand side of the integral equation under the assumption that $\Gamma$ is a straight line or an arc of the unit circle.

Example 6. Let $\Gamma$ be a segment of the straight line:

$$
\Gamma=\{(x, 0):-1 \leq x \leq 1\},
$$

and the parameters $f \equiv 1, \beta \equiv 1$. The corresponding exact solution of integral equation (22) can be written as

$$
\mu(s)=-\frac{2}{1+\beta^{2}} \frac{s}{\sqrt{1-s^{2}}} .
$$

The solution of problem (2) can be expressed by (11). The exact analytical expressions for potentials under the assumption are

$$
\begin{aligned}
& w[\mu](x)=\frac{\sqrt{r_{1} r_{2}}}{1+\beta^{2}}\left\{\cos \frac{\varphi_{1}}{2} \cos \frac{\varphi_{2}}{2}\right. \\
& \left.-\sin \frac{\varphi_{1}}{2} \sin \frac{\varphi_{2}}{2}\right\}-\frac{x_{1} \sqrt{r_{1} r_{2}}}{1+\beta^{2}}, \\
& v[\mu](x)=\frac{\sqrt{r_{1} r_{2}}}{1+\beta^{2}}\left\{\cos \frac{\varphi_{1}}{2} \sin \frac{\varphi_{2}}{2}\right. \\
& \left.+\sin \frac{\varphi_{1}}{2} \cos \frac{\varphi_{2}}{2}\right\}-\frac{x_{2} \sqrt{r_{1} r_{2}}}{1+\beta^{2}},
\end{aligned}
$$

where $r_{1}=\sqrt{\left(x_{1}+1\right)^{2}+x_{2}^{2}}, r_{2}=\sqrt{\left(x_{1}-1\right)^{2}+x_{2}^{2}}, \varphi_{1}=$ $\arctan \left(x_{2} /\left(x_{1}+1\right)\right), \varphi_{2}=\arctan \left(x_{2} /\left(x_{1}-1\right)\right)$.

Now, let us consider the numerical solution at this time. Obviously,

$$
\frac{\sin \varphi_{0}(y(s), y(\sigma))}{|y(s)-y(\sigma)|}-\frac{1}{(\sigma-s)|\dot{y}(\sigma)|}=0
$$

The spectral Galerkin approximate solution of $\mu$ is equal to the exact solution.

This situation allows us to test the accuracy of the numerical method of potentials. We choose

$$
W=\{(x, d):-1 \leq x \leq 1\}
$$

as the points where we compute the potentials. $L^{2}$-errors are listed in Table 1, where the numerical solutions $\widetilde{w}, \widetilde{v}$ are compared with analytical solutions $w, v$. Table 1 also contains the $L^{2}$-errors of the potentials $W, V$ which are computed by the methods in [11].

It can be seen that the computation errors of the potentials is on the good control. The errors increase as $d$ decreases, because logarithmic function in $(50)$ and $V(x, y)$ in (51) have singularities when $d$ is very small.

As shown in Table 1, the numbers of single-potential are superior to those in [11] when $d>10^{-3}$, but inferior to those when $d=10^{-3}$. That is because (50) is not as accurate as the approximation formula in [11] when $d$ is very small. But it is 
TABLE 6: $L^{2}$-errors and relative $L^{2}$-errors of an arc on the unit circle with $f \equiv 1$.

\begin{tabular}{lllll}
\hline$N$ & $\|w[\mu]-\widetilde{w}[\mu]\|_{L^{2}}$ & $\frac{\|w[\mu]-\widetilde{w}[\mu]\|_{L^{2}}}{\|w\|_{L^{2}}}$ & $\|v[\mu]-\widetilde{v}[\mu]\|_{L^{2}}$ & $\frac{\|v[\mu]-\widetilde{v}[\mu]\|_{L^{2}}}{\|v\|_{L^{2}}}$ \\
\hline 3 & $6.0414 \times 10^{-7}$ & $1.3580 \times 10^{-6}$ & $5.8808 \times 10^{-7}$ & $1.3858 \times 10^{-6}$ \\
5 & $4.1341 \times 10^{-9}$ & $9.2929 \times 10^{-9}$ & $3.9567 \times 10^{-9}$ & $9.3237 \times 10^{-9}$ \\
10 & $2.1323 \times 10^{-13}$ & $4.7930 \times 10^{-13}$ & $1.9174 \times 10^{-13}$ & $4.5181 \times 10^{-13}$ \\
\hline
\end{tabular}

TABLE 7: $L^{2}$-errors and relative $L^{2}$-errors of an arc on the unit circle with $f(x)=\sin (x)+\cos (x)$.

\begin{tabular}{lcccc}
\hline$N$ & $\|w[\mu]-\widetilde{w}[\mu]\|_{L^{2}}$ & $\frac{\|w[\mu]-\widetilde{w}[\mu]\|_{L^{2}}}{\|w\|_{L^{2}}}$ & $\|v[\mu]-\widetilde{v}[\mu]\|_{L^{2}}$ & $\frac{\|v[\mu]-\widetilde{v}[\mu]\|_{L^{2}}}{\|v\|_{L^{2}}}$ \\
\hline 3 & $2.2349 \times 10^{-3}$ & $5.6730 \times 10^{-3}$ & $2.3333 \times 10^{-3}$ & $5.8689 \times 10^{-3}$ \\
5 & $1.9499 \times 10^{-5}$ & $4.7093 \times 10^{-5}$ & $1.8751 \times 10^{-5}$ & $4.7164 \times 10^{-5}$ \\
10 & $9.7051 \times 10^{-12}$ & $2.3439 \times 10^{-11}$ & $1.0428 \times 10^{-11}$ & $2.6228 \times 10^{-11}$ \\
\hline
\end{tabular}

adequate enough for our purpose and our formula is easier to implement.

According to Table 1, our results of angular potential have higher accuracy than results of [11]. In [11], the author subdivides each interval of length $h$ into some subintervals uniformly in order to improve the accuracy. The values of $\mu$ on subintervals are computed by interpolation. In this paper, we need to increase $J$ so that to decrease the errors. The potential can be evaluated easily without interpolation since the numerical result of $\mu$ is a function. The interpolation procedure may deduced the related accuracy.

The straight-line case is too special to show the accuracy of the method for arbitrary arcs. Therefor we consider further numerical tests on a curved arc.

Example 7. Suppose $\Gamma$ is an arc of the unit circle

$$
\Gamma=\{(\cos x, \sin x):-1 \leq x \leq 1\} .
$$

If $f \equiv 1$, then the density function can be given by

$$
\mu(x)=\frac{-2}{\left(1+\beta^{2}\right) \sqrt{\sin ((x+1) / 2) \sin ((1-x) / 2)}} \sin \frac{x}{2} .
$$

If $f(x)=\cos x+\sin x$, the exact density function is

$$
\begin{aligned}
\mu(x)= & \frac{-2}{\left(1+\beta^{2}\right) \sqrt{\sin ((x+1) / 2) \sin ((1-x) / 2)}} \\
& \times\left[\sin \frac{x}{2} \cos (-1)-\sin \frac{3 x}{2}\right. \\
& \left.\quad+\cos \frac{3 x}{2}-\cos \frac{x}{2} \cos (-1)\right] .
\end{aligned}
$$

The potentials under these two assumptions cannot be given by explicit expressions. The comparison between numerical solution $\widetilde{M}$ and the analytical solution $\mu$ is not convenient because of the space $\widetilde{H}^{-1 / 2}(\Gamma)$ they belong to. Let $\widetilde{M}_{*}=$ $\sqrt{1-x^{2}} \widetilde{M}, \mu_{*}=\sqrt{1-x^{2}} \mu . L^{2}$-errors between $\widetilde{M}_{*}$ and $\mu_{*}$ are listed in Tables 2 and 4 . We observe excellent agreement between them. The accuracy of $\widetilde{M}_{*}$ increases if $N$ increases. But no significant change can be seen whatever $J$ increases or not. The numerical solution $\widetilde{\mu}_{*}$ of [11] is compared with the analytical solution $\mu_{*}$ in Tables 3 and 5 . Results in Tables 2 and 4 are smaller than those in Tables 3 and 5. The computational schemes in this paper are stable and efficient.

Let $\widetilde{w}[\mu], \widetilde{v}[\mu]$ be the angular potential and singlepotential computed by (50) and (51). We use the exact density function to get them. Let $\widetilde{w}[\widetilde{\mu}], \widetilde{v}[\widetilde{\mu}]$ be the angular potential and single-potential used by numerical approximation of the density function.

We compute the potentials in a parallel surface of $\Gamma$ defined by

$$
W=\{(d \cos x, d \sin x),-1 \leq x \leq 1\},
$$

where $d=1.01$. Results of the tests are presented in Tables 6 and 7. Obviously, errors decrease as $N$ increases. When $f \equiv$ 1 , it can be seen that the approximation is satisfactory even when $N=3$. The simulation of $f=\cos x+\sin x$ is not as good as the simulation of $f \equiv 1$. The errors when $N=5$ with $f=\sin x+\cos x$ are almost the same as the errors when $N=3$ under the condition of $f \equiv 1$. But the accuracy for both cases is high, when $N=10$.

Example 8. In this example, we take the arc

$$
y=0.2 x^{2}-0.1 x+0.3
$$

and the parameters

$$
\begin{aligned}
\beta=1, \quad f= & \frac{1}{\sqrt{1+0.4 x-0.1}} \\
& \times\left(0.16 x^{3}+1.08 x^{2}-2.94 x+2.74\right) .
\end{aligned}
$$

We compute the single-potential and angular potential on a semicircular arc:

$$
(2 \cos (t), 2 \sin (t)), \quad 0 \leq t \leq \pi,
$$




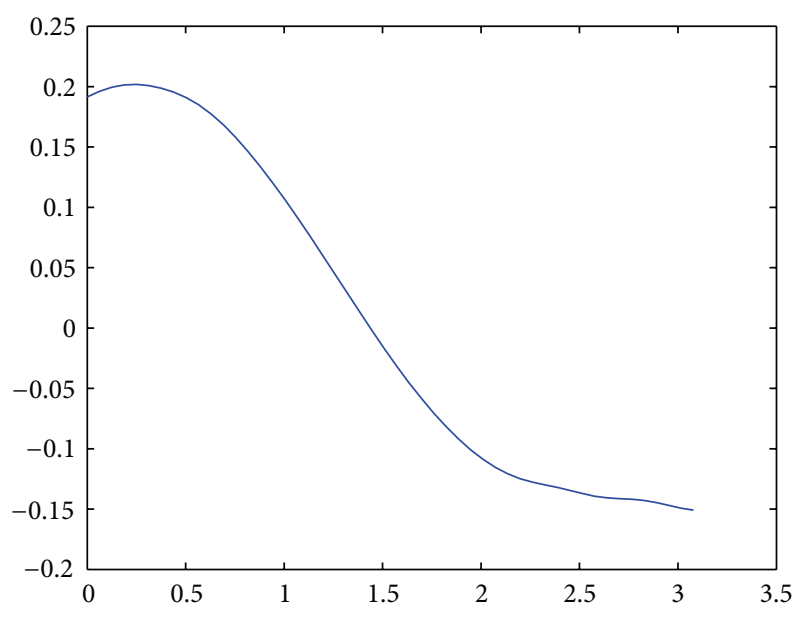

FIGURE 1: Single-layer potential on a semicircular arc.

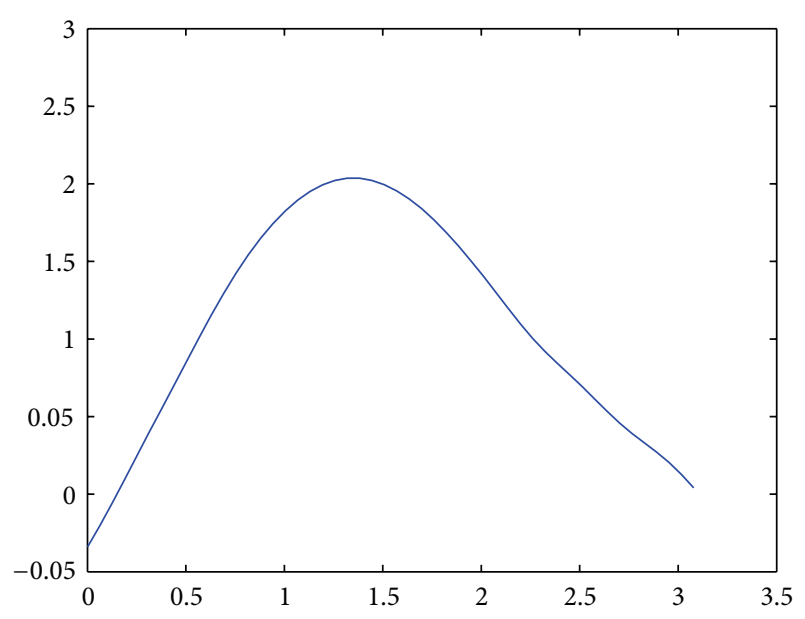

FIgURE 2: angular potential on a semicircular arc.

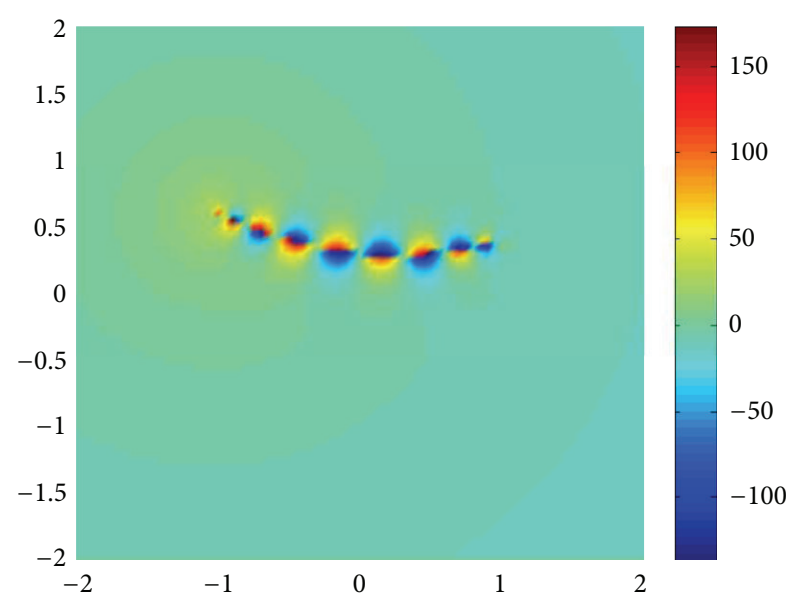

FIGURE 3: Electric-field potential $u$. see Figures 1 and 2. We choose $J=160$ and $N=10$ to compute the electric-field potential $u$ which is shown in Figure 3.

\section{Conclusions}

In this paper, we have demonstrated an efficient and highly accurate boundary Galerkin method for a skew-derivative problem. The problem is considered in Sobolev spaces firstly. In our method, approximate calculation of singular integral can be avoided. The density function can be evaluated at any point without interpolation once the series coefficients have been determined. It is direct, simple, and fast.

\section{Acknowledgment}

The work of the first author is supported by the Youth Natural Science Foundation of Heilongjiang (Grant no. QC2011c103).

\section{References}

[1] S. A. Yang, "A numerical method for scattering from acoustically soft and hard thin bodies in two dimensions," Journal of Sound and Vibration, vol. 250, no. 5, pp. 773-793, 2002.

[2] R. Kress and K.-M. Lee, "Integral equation methods for scattering from an impedance crack," Journal of Computational and Applied Mathematics, vol. 161, no. 1, pp. 161-177, 2003.

[3] C. Jerez-Hanckes and J. C. Nedelec, "Hybrid Galerkin method for elliptic and wave propagation problems in $\mathbb{R}^{3}$ over planar structures," in Integral Methods in Science and Engineering, vol. 2, chapter 19, Computational Methods, 2009.

[4] F. Cakoni and D. Colton, "The linear sampling method for cracks," Inverse Problems, vol. 19, no. 2, pp. 279-295, 2003.

[5] P. A. Krutitskii, N. C. Krutitskaya, and G. Y. Malysheva, "A problem related to the hall effect in a semiconductor with an electrode of an arbitrary shape," Mathematical Problems in Engineering, vol. 5, no. 1, pp. 83-95, 1999.

[6] P. A. Krutitski1̌, "The Dirichlet problem for the Helmholtz equation in the exterior of cuts in the plane," Computational Mathematics and Mathematical Physics, vol. 34, no. 8-9, pp. 1237-1258, 1994.

[7] P. A. Krutitskii and V. V. Kolybasova, "A generalization of the Neumann problem for the Helmholtz equation outside cuts on the plane," Differential Equations, vol. 41, no. 9, pp. 1213-1224, 2005.

[8] P. A. Krutitskiü, "On the properties of solutions of the Dirichlet problem for the Helmholtz equation in a two-dimensional domain with cuts," Differential Equations, vol. 43, no. 9, pp. 1200-1212, 2007.

[9] P. A. Krutitskiü, "A boundary value problem for the Laplace equation outside cuts on the plane with different conditions of the third kind on opposite sides of the cuts," Differential Equations, vol. 45, no. 1, pp. 86-100, 2009.

[10] J. Liu, P. A. Krutitskii, and M. Sini, "Numerical solution of the scattering problem for acoustic waves by a two-sided crack in 2dimensional space," Journal of Computational Mathematics, vol. 29, no. 2, pp. 141-166, 2011.

[11] P. A. Krutitskii, D. Y. Kwak, and Y. K. Hyon, "Numerical treatment of a skew-derivative problem for the Laplace equation in 
the exterior of an open arc," Journal of Engineering Mathematics, vol. 59, no. 1, pp. 25-60, 2007.

[12] H. Ammari, J. Garnier, H. Kang, W.-K. Park, and K. Sølna, "Imaging schemes for perfectly conducting cracks," SIAM Journal on Applied Mathematics, vol. 71, no. 1, pp. 68-91, 2011.

[13] H. Ammari, H. Kang, and H. Lee, Layer Potential Techniques in Spectral Analysis, vol. 153, American Mathematical Society, Providence, RI, USA, 2009.

[14] H. Ammari, H. Kang, H. Lee, and W.-K. Park, "Asymptotic imaging of perfectly conducting cracks," SIAM Journal on Scientific Computing, vol. 32, no. 2, pp. 894-922, 2010.

[15] W. McLean, Strongly Elliptic Systems and Boundary Integral Equations, Cambridge University Press, Cambridge, UK, 2000.

[16] G. C. Hsiao and W. L. Wendland, Boundary Integral Equations, vol. 164 of Applied Mathematical Sciences, Springer, Berlin, Germany, 2008.

[17] R. Li, The Galerkin Method of Boundary Value Problem, Shanghai Scitific and Technical Publishers Press, 1988. 


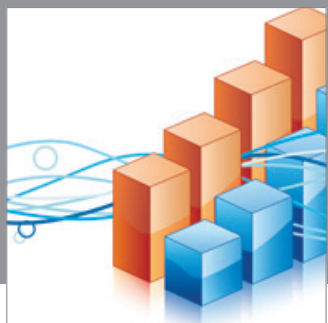

Advances in

Operations Research

mansans

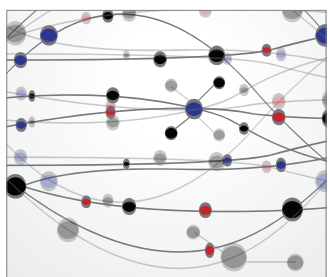

The Scientific World Journal
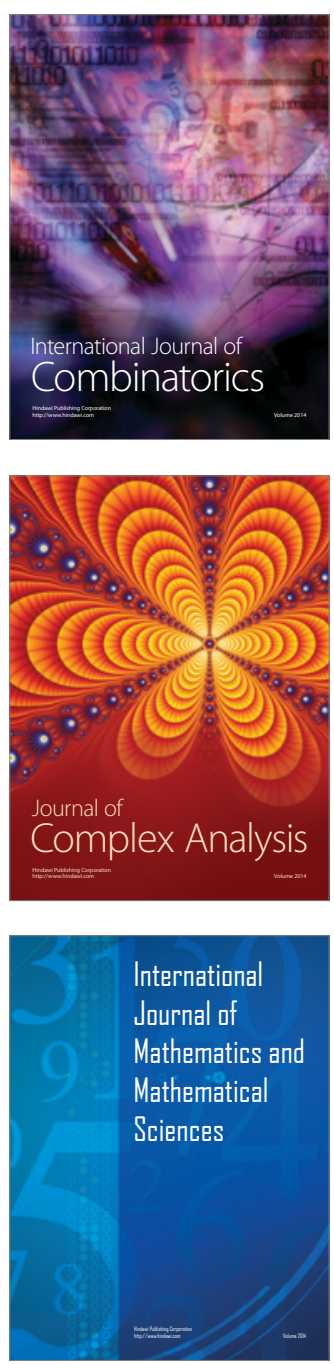
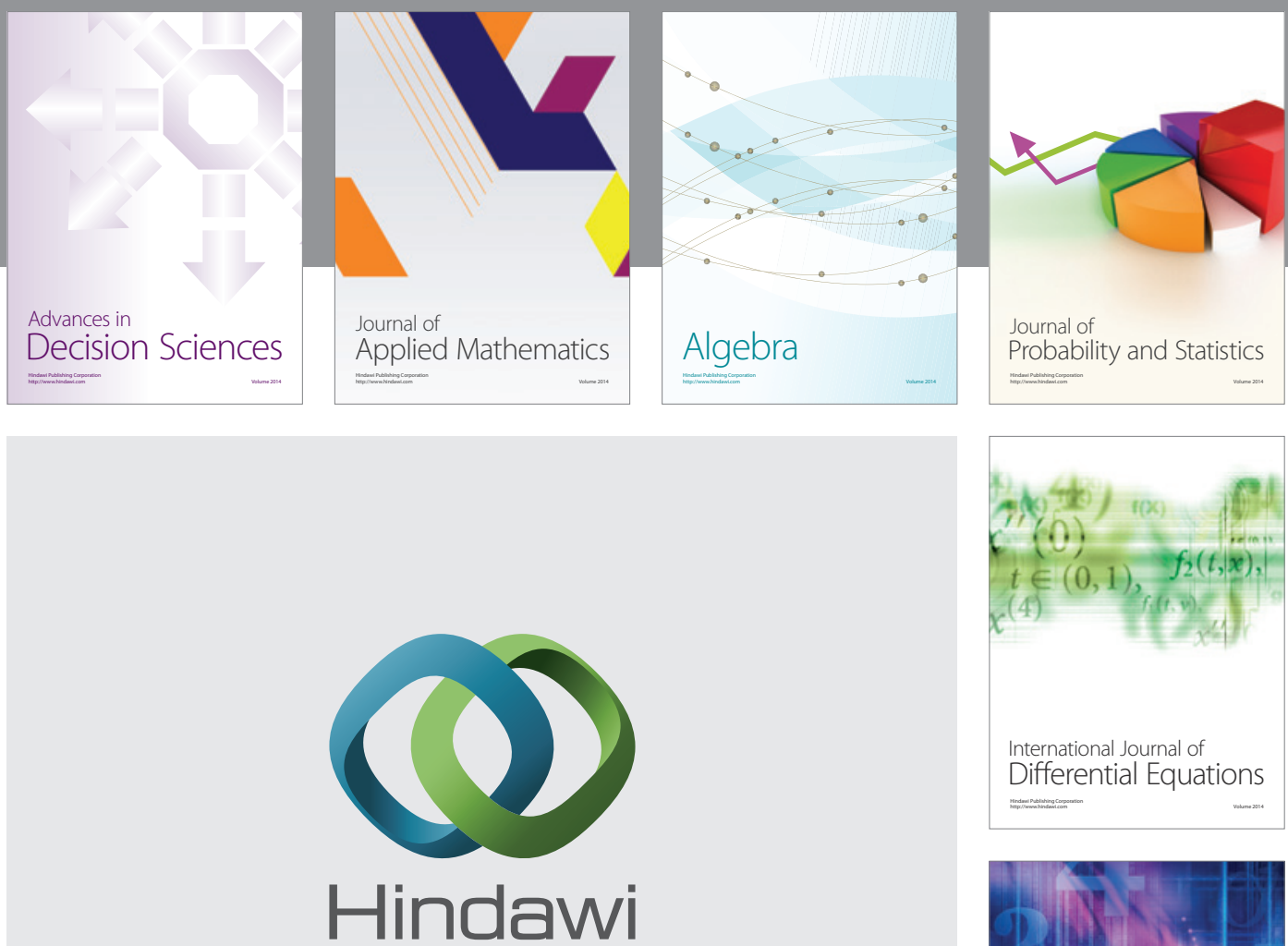

Submit your manuscripts at http://www.hindawi.com
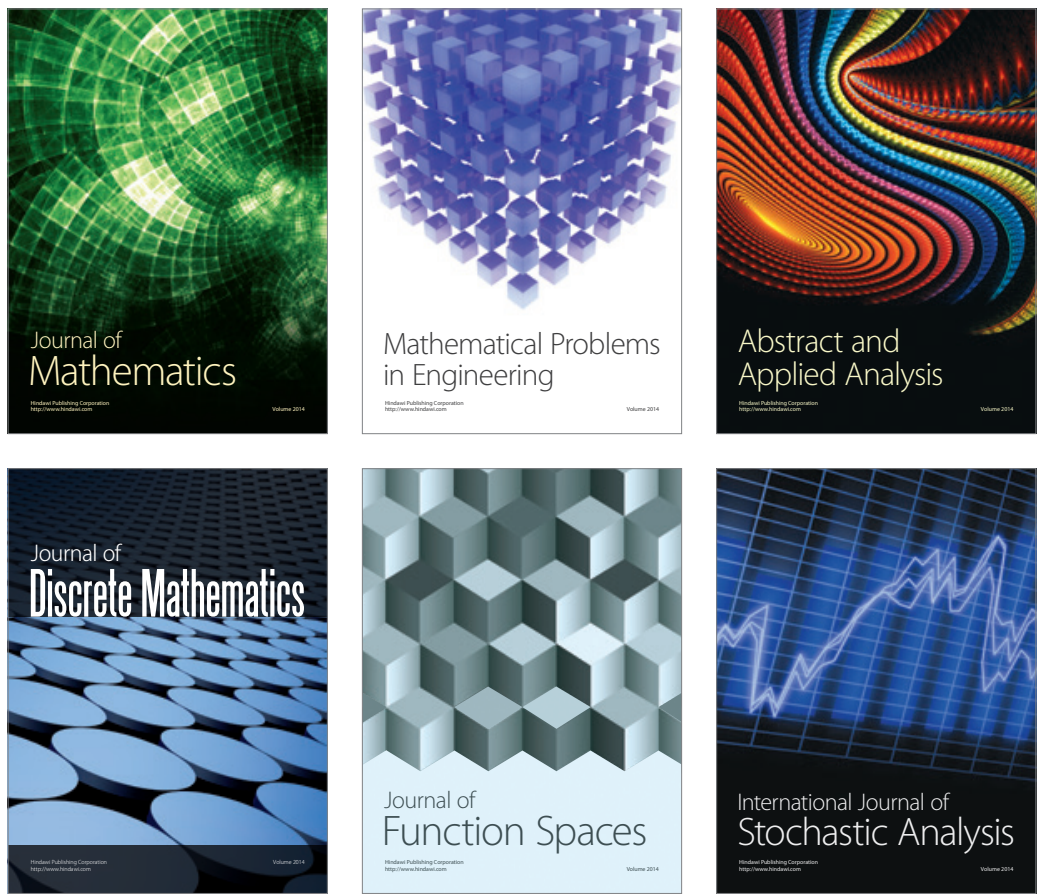

Journal of

Function Spaces

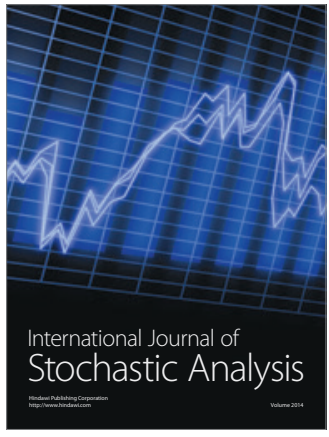

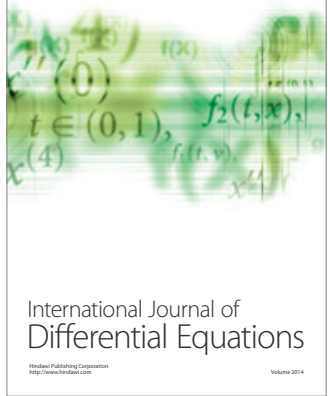
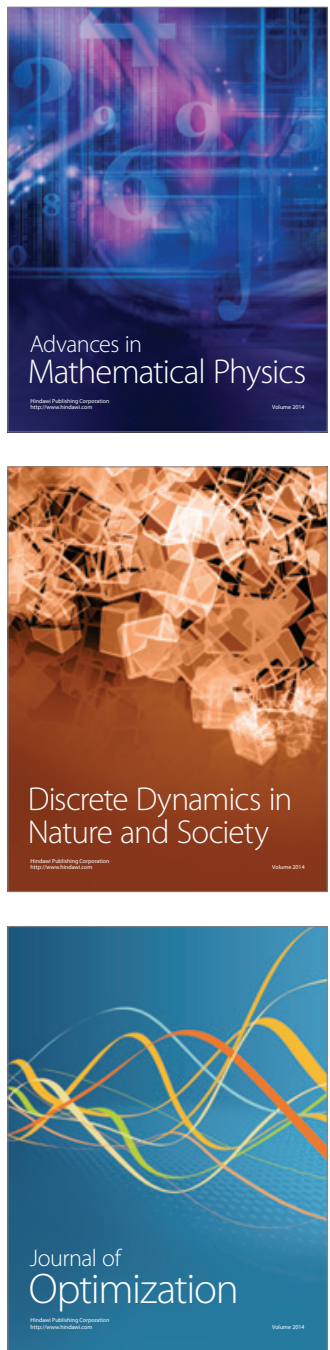